\title{
Backlight Dimming in Power-Aware Mobile Displays
}

\author{
Ali Iranli \\ University of Southern California \\ iranli@usc.edu
}

\author{
Wonbok Lee \\ University of Southern California \\ wonbokle@usc.edu
}

\author{
Massoud Pedram \\ University of Southern California \\ pedram@usc.edu
}

\begin{abstract}
This paper presents a temporally-aware backlight scaling (TABS) technique for video streams. The goal is to maximize energy saving in the display system by means of dynamic backlight dimming subject to a user-specified tolerance on the video distortion. The video distortion itself comprises of (i) an intraframe (spatial) distortion component due to frame-sensitive backlight scaling and transmittance function tuning and (ii) an inter-frame (temporal) distortion component due to large-step backlight dimming across multiple frames and modulated by the physiological characteristics of the human visual system. The proposed backlight scaling technique is capable of efficiently computing the flickering effect online and subsequently using a measure of the temporal distortion to appropriately adjust the slack on the intra-frame spatial distortion. The proposed technique has been implemented on the Apollo Testbed II hardware platform. Actual current measurements on this platform demonstrate the superiority of TABS compared to previous backlight dimming techniques.
\end{abstract}

\section{Categories and Subject Descriptors}

B.4.2. [Input/Output Devices]: Image display

\section{General Terms}

Algorithms, Human Factors, Measurement

\section{Keywords}

LCDs, Backlight scaling, Human Visual System

\section{INTRODUCTION}

Major sources of power dissipation in a portable electronic device are many and vary as a function of the device functionality and performance specification. In reality, many of these devices are equipped with an Active Matrix Liquid Crystal Display (LCD), which tends to account for a significant portion of the total system power. These LCD displays require two power sources, a DC-AC inverter to power the CCFL used as the backlight and DC-DC converters needed to boost and drive the rows and columns of the LCD panel. Power is also consumed in the bus interface, analogdigital converter, LCD controller circuit, watchdog timer, RAM array, etc. Among these sources of power consumption, the display backlight is the single largest power consumer in a typical LCD module. Unlike the other system resources, one cannot tackle this power thirsty component with some form of power shutdown.

There are two main techniques for lowering the power consumption of LCD module. The first class has focused on the digital/analog interface between the graphics controller and the LCD controller. These techniques try to minimize the energy

Permission to make digital or hard copies of all or part of this work for personal or classroom use is granted without fee provided that copies are not made or distributed for profit or commercial advantage and that copies bear this notice and the full citation on the first page. To copy otherwise, or republish, to post on servers or to redistribute to lists, requires prior specific permission and/or a fee.

DAC 2006, July 24-28, 2006, San Francisco, California, USA.

Copyright 2006 ACM 1-59593-381-6/06/0007 ...\$5.00. consumption by taking advantage of different encoding schemes to minimize the switching activity of the electrical bus. The second class has focused on the video controller and the backlight source. The key intuition is that the human eye's perception of the light, which is emitted from the LCD panel, is a function of two parameters, 1) intensity of the backlight and 2) transmittance of pixels in the LCD panel. Since the variation in power consumption of the backlight lamp for different output luminance values is orders of magnitude larger than power consumption of the LCD panel for different pixel values, one can save energy achieve the same perception in human eyes by simply dimming the backlight and increasing the LCD transmittance.

In their pioneering work [1], Chang et al. proposed a Dynamic backlight Luminance Scaling technique (DLS) to reduce the energy consumption of the LCD displays. This approach has two drawbacks, a) it manipulates every pixel on the screen oneby-one limiting the application of this approach to still images or low-frame-rate videos; b) it achieves energy saving at the cost of loss in visual information. Reference [2] improved this approach by eliminating the pixel-by-pixel transformation of the displayed image through minor hardware modifications to the built-in LCD reference driver. Reference [3] proposed a method for image transformation, whereby the dynamic range of the original image is reduced such that the incurred image distortion is no more than a pre-specified value. Reference [4] presented a backlight scaling technique, which is based on a tone reproduction operator.

Although the aforesaid techniques are effective backlight scaling approaches, they all suffer from a common shortcoming, i.e., temporal blindness. They apply the backlight scaling scheme to each frame of a video sequence individually without considering flickering effects, which may result as a consequence of frequent and abrupt changes in the backlight intensity. In the remainder of this paper, we will refer to all of the prior techniques for video as Frame-sensitive Backlight Scaling (FBS) techniques.

In contrast, this paper proposes a backlight scaling scheme which is sensitive to the spatiotemporal information of video sequence. The key idea is to decompose the maximum allowed distortion between the original video and the backlight scaled one into two components, 1) the spatial distortion which is the intraframe luminance distortion between the respective frames of the original and backlight scaled video sequences, and 2) the temporal distortion which is the inter-frame distortion due to (large-scale) changes of luminance over time when comparing the original and backlight scaled video sequences. We take advantage of an analytical model of the Critical Fusion Frequency (CFF) and the temporal information about the average luminance of each frame. Next, we limit the maximum allowed spatial distortion that the FBS scheme create, and therefore, limit the overall maximum video distortion below a certain user specified limit.

This paper provides:

1. A useful characterization of video distortion, which accounts for both spatial and temporal components of video distortion and uses an efficient model of the HVS. 
2. The first temporally-aware backlight scaling technique for high frame-rate video, which maximizes the backlight dimming-driven energy savings in the LCD module subject to a bound on tolerable video distortion.

3. Efficient hardware realization of the proposed temporally-aware backlight scaling.

In the following, the basic background on the temporal response of human visual system and prior work of dynamic backlight scaling will be discussed. Next, in section 3 temporallyaware backlight scaling approach will be explained. Sections 4 and 5 will provide the experimental results and conclusions.

\section{BACKGROUND}

\subsection{Temporal Response of Human Visual System}

The Human Visual System (HVS) functions at a wide range of light levels, from starlight to bright sunlight. To cope with this enormous range, various processes of light adaptation have evolved. Light adaptation refers to the changes in our sensitivity to light, which allow us to adapt to a wide range of light levels. These adjustments do not simply modify our sensitivity to light; they change the way our visual system processes the spatial and temporal variations.

In the spatial domain, with increases in ambient light level, mechanisms of adaptation come into play. A fundamental measure of field adaptation is the Threshold Versus Intensity (TVI) function that is a plot of the intensity needed to detect a test light as a function of the intensity of the background upon which it is presented.[8] This notion was exploited in histogram equalization for backlight scaling technique (HEBS) [3].

On the other hand, in the temporal domain, studies of the dynamics of light perception can be divided into two categories based on the stimulation method utilized for the HVS characterization. The first category is based on aperiodic stimuli, which tries to measure the impulse response of HVS. In these experiments, the sensitivity of the HVS is measured when a brief test light is presented to the HVS before, during, and after the presentation of a much stronger background light. [5]. The second category tries to measure the Critical Fusion Frequency (CFF) of the HVS at various amplitude sensitivity values (AS values.) The CFF is the minimum frequency, $f^{*}$, above which the observer cannot detect any flickering effect when a series of light flashes at that frequency is presented to him/her. The AS at frequency $f^{*}$ is the ratio of the minimum required amplitude of the flash light at frequency $f^{*}$ to the average ambient luminance such that the flickering effect is perceived [5].

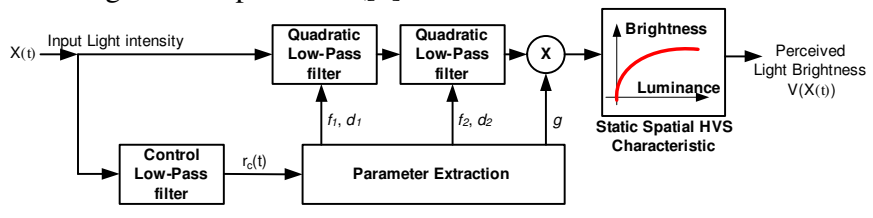

Figure 1. Temporal model of HVS

In this paper, we adopt a computational temporal response model of the HVS of Weigand et al. [6] (cf. Figure 1.) This model can be used to determine the AS threshold of an observer when presented with varying light intensities. Note that the input to this model is a scalar value representing the luminance of the viewing area, whereas the output is the intensity of perceived luminance. If the difference between the DC value and the amplitude of a given frequency, $f^{*}$, in the output of the model exceeds a predefined threshold $\delta$, then the flickering is perceived.

Figure 2 shows a typical frequency domain transfer function of this model. Note that for a fixed DC value, i.e. fixed background luminance, as we increase the frequency of the flickering light the required amplitude for the flickering to be perceived is almost constant for frequencies below $10 \mathrm{~Hz}$ and then increases exponentially. Moreover, for a given frequency of flashing light by increasing the background luminance, the minimum amplitude of the flashing light for which the flickering is perceived decreases.

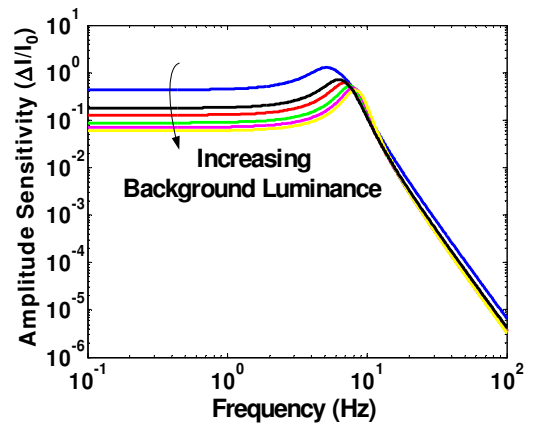

Figure 2. Sample amplitude sensitivity of HVS for sinusoidal input with varying $\mathrm{DC}$ value

\subsection{Previous Backlight Scaling}

Mathematically, in a transmissive TFT-LCD monitor, for a pixel with value $x$, the luminance $L(x)$ of the pixel is:

$$
L(x)=b . t(x)
$$

where $t(x)$ is the transmissivity of the TFT-LCD cell for pixel value $x$, and $b \in[0,1]$ is the (normalized) backlight illumination factor with $b=1$ representing the maximum backlight illumination and $b=0$ representing no backlight. Note that $t(x)$ is a linear mapping from $[0,255]$ domain to $[0,1]$ range.

The key idea of backlight scaling technique is to dim the backlight and compensate for the luminance loss by adjusting the grayscale of the image to increase its brightness or contrast. More precisely,

$$
L(x)=\beta . t(\Phi(x, \beta))
$$

where $0<\beta \leq 1$ is the backlight scaling factor and $\Phi(X, \beta)$ is the pixel transformation function.

Reference [2] proposed a method in which the pixel values in both dark and bright regions of the image are used to enable a more aggressive backlight dimming while maintaining the contrast fidelity of the image. The key idea is to first truncate the image histogram on both ends to obtain a smaller dynamic range for the image pixel values and then to spread out the pixel values in this range (by applying an affine transformation). Reference [3] proposed a method for image transformation, whereby the dynamic range of the original image is reduced such that the incurred image distortion is no more than a pre-specified value. The method, however, had two disadvantages; a) the distortion characterization curve was dependent on the displayed image; b) the equalization algorithm required histogram information of the displayed image to calculate the image transformation function.

The aforementioned techniques are effective for still images; however, if one tries to apply these techniques on a per-frame 
basis to a video stream, the output video quality can significantly be degraded. This is because different frames in a video clip may have different characteristics, and therefore, FBS may result in different pixel transformation functions $(\Phi)$, and thus, different backlight scaling factors $(\beta)$. The abrupt changes in $\Phi$ and $\beta$ can subsequently result in a large flickering effect over time.

\section{THE PROPOSED APPROACH}

In FBS techniques, the spatial distortion is upper-bounded by a user specified maximum allowed value (cf. Figure 3 ). The second component of distortion is the changes in the luminance of the backlight-scaled video compared to the original video, i.e. the temporal distortion.

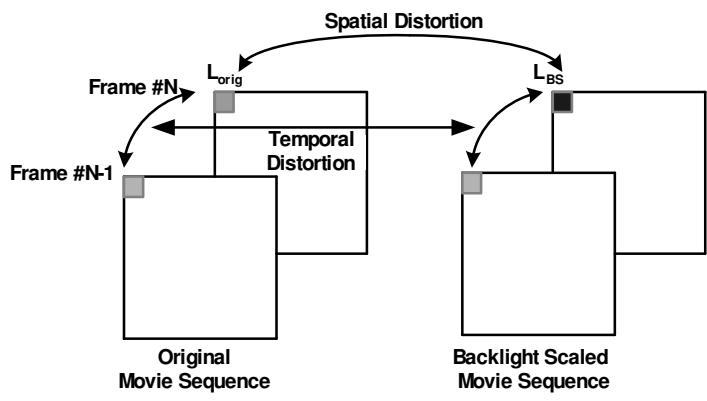

Figure 3. Spatial vs. Temporal distortions

Let $\mathcal{X}$ and $\mathfrak{y}$ denote the original and the backlight scaled versions of a video sequence with total number of frames, $N$. We define the distortion between two video sequences $\mathcal{X}$ and $\mathcal{U}$ as,

$$
\begin{aligned}
& D(\mathrm{X}, \mathrm{Y})=\alpha \cdot D_{s p t}(\mathrm{X}, \mathrm{Y})+(1-\alpha) \cdot D_{t m p}(\mathrm{X}, \mathrm{Y}) \text { where } \\
& D_{s p t}(\mathrm{X}, \mathrm{Y})=\max _{i}\left(D_{s p t}\left(\mathrm{X}, \mathrm{Y}_{i}\right)\right) \\
& D_{t m p}(\mathrm{X}, \mathrm{Y})=\frac{\sum_{j}\left(\mathrm{~F}\{V(\mathrm{X})\}_{j}-\mathrm{F}\{V(\mathrm{Y})\}_{j}\right)^{2}}{\sum_{j}\left(\mathrm{~F}\{V(\mathrm{X})\}_{j}\right)^{2}}
\end{aligned}
$$

where $D_{s p t}(\mathcal{X}, \mathfrak{y})$ is the spatial distortion between still images, $¥$ and $\mathfrak{U} ; V($.$) is the perceived light intensity when an input is given$ to the HVS (cf. Figure 1), and $\mathfrak{F}\{$.$\} is the Fourier transform$ operator. Finally, $\alpha$ is the weighting coefficient. First term in equation (3) captures the spatial distortion between the respective frames of video sequences $\mathbb{X}$ and while the second term is the mean square error between the spectral power density of the original video sequence and the backlight scaled one.

Note that equation (3) is hard to evaluate because the first term is in time domain while the second term is defined in the frequency domain. To circumvent this problem, equation (3) can be simplified by using the Parseval's theorem, which simply states that integral of squared signal is equal to integral of its spectral power density, therefore,

$$
D_{\text {trp }}(\mathrm{X}, \mathrm{Y})=1+\frac{\frac{1}{N} \sum_{j}\left(V\left(\mathrm{Y}_{j}\right)\right)^{2}-2 V\left(\mathrm{X}_{0}\right) \cdot V\left(\mathrm{Y}_{0}\right)}{\frac{1}{N} \sum_{j}\left(V\left(\mathrm{X}_{j}\right)\right)^{2}}
$$

Figure 4 shows the block diagram of this approach. The key idea is to measure the temporal distortion of backlight scaled video and then feedback this information to dynamically change the maximum allowed spatial distortion.

To measure the temporal distortion, $D_{\text {tmp }}$, of the video sequence we use the following procedure,
1. For each original frame, $\mathfrak{X}_{t}$, and backlight scaled frame, $\mathfrak{W}_{t}$, at time $t$, calculate the mean brightness value, $\bar{X}(t)$ and $\bar{Y}(t)$ of all pixels in the respective frames.

2. Filter signals $\bar{X}(\mathrm{t})$ and $\bar{Y}(\mathrm{t})$, using the temporal response model of HVS to get perceived luminance signals $\bar{X}_{\mathrm{HVS}}(\mathrm{t})$ and $\bar{Y}_{\mathrm{HVS}}(\mathrm{t})$, respectively.

3. Calculate equation (4) for $\bar{X}_{\mathrm{HVS}}(\mathrm{t})$ and $\bar{Y}_{\mathrm{HVS}}(\mathrm{t})$ to get $D_{\mathrm{tmp}}$. Next, we use $D_{\text {tmp }}$ to modify the maximum allowed spatial distortion, $D_{\mathrm{spt}}^{\max }$, in FBS techniques.

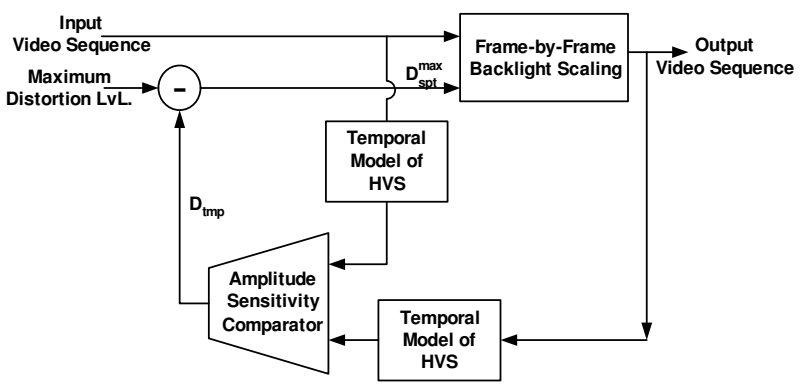

Figure 4. Temporally-aware backlight scaling

\section{IMPLEMENTATION \& RESULTS}

To assess the effectiveness of TABS, we used the experimental setup shown in Figure 5. The demonstration platform [7], Apollo Test-bed II, uses Intel X-scale 80200 processor and includes a 6.4 " LCD at $640 * 480$ resolution. We modified the MPEG-1 decoder application to incorporate the FBS technique of [3], which from now on will be referred to as HEBS (as the authors intended.)

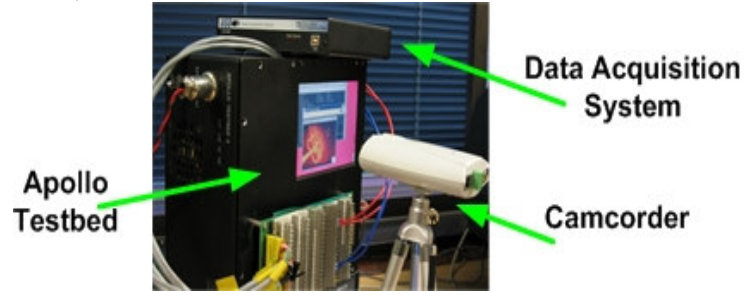

Figure 5. The experimental Setup

As described in [1], the control low pass filter is a first order filter with following transfer function,

$$
H_{C-L P}(f)=\frac{1}{j 2 \pi(1.59 f)+1}
$$

whereas the cascade of two quadratic low pass filters has the general transfer function of,

$$
H_{L P}(f)=\left[\frac{f_{1}^{2}}{f_{1}^{2}+j d_{1} f_{1} f-f^{2}}\right] \cdot\left[\frac{f_{2}^{2}}{f_{2}^{2}+j d_{2} f_{2} f-f^{2}}\right]
$$

with parameters given by,

$$
\begin{aligned}
& z_{c}(t)=1 /\left[1+\left(r_{c}(t) / 138.839\right)^{0.5}\right] \\
& f_{1}(t)=-4.299 \cdot z_{c}(t)+11.65 \\
& f_{2}(t)=-24.36 \cdot z_{c}(t)+28.68 \\
& d_{1}(t)=-1.218 \cdot z_{c}(t)+0.616 \\
& d_{2}(t)=-1.003 \cdot z_{c}(t)+0.448
\end{aligned}
$$

Finally, the controllable gain is given by,

$$
g=2.2 \cdot\left[\left(45.899+r_{c}(t)\right)^{-0.641}\right] \cdot\left[\left(0.001+r_{c}(t)\right)^{-0.5114}\right]
$$


where $r_{c}(t)$ is the control signal at the output of the control low pass filter given in equation (5-a) (cf. Figure 1).

To implement TABS, we followed two steps: 1) The HVS model presented in section 2 is a continuous-time model while the average luminance of each frame in the displayed video is calculated on a frame by frame basis resulting in a discrete-time signal. Therefore, we transformed the continuous-time filters given by equations $(5-\mathrm{a}, \mathrm{d})$ to discrete-time filters. 2) The output of the controller low-pass filter in Figure $\mathbf{1}$ does not change significantly. This is due to limited range of output luminance values for typical LCD. Therefore, we assumed that the output of control filter is a constant, i.e., $r \mathrm{c}(t)=r 0$ (cf. Figure 1). This assumption results in fixed quadratic filters and gain blocks in the temporal model of the HVS.

From these observations and by calculating output of the control low-pass filter, $r_{0}$, we obtain the following digital filter,

$$
H_{L P}(f)=\left[\frac{0.017}{1.067-2.05 z+z^{2}}\right] \cdot\left[\frac{0.011}{1.05+-2.03 z+z^{2}}\right]
$$

Subsequently, we implemented this digital filter and calculated the output of the HVS model for the average luminance value of each frame in the video sequence.
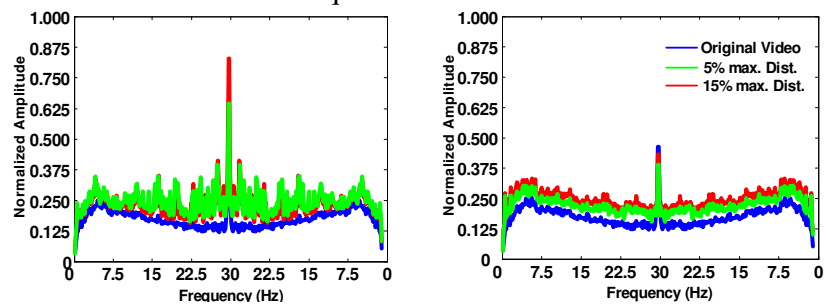

A little mermaid
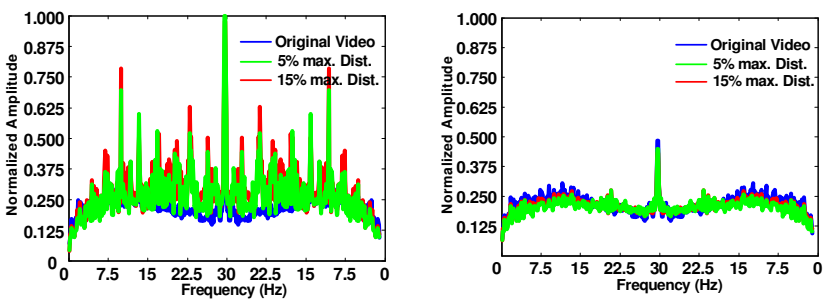

The incredibles

\section{a. backlight scaling by HEBS b. backlight scaling by TABS}

Figure 6. Fourier transform of output video sequence

For the online calculation of the temporal distortion, $D_{t m p}$, we approximated the calculation in equation (4) by averaging the signal over a fixed number of previously filtered average luminance values i.e., we used a moving average technique. Next, we used this moving average value and the maximum allowed video distortion, $D_{\max }$, in equation (3) to calculate the maximum allowed spatial distortion, $D_{s p t}^{\max }$. Finally, we used $D_{s p t}^{\max }$ as the distortion bound in HEBS.

To measure the temporal distortion of the backlight and the pixel transformation function, we calculated the average luminance value for each of the recorded frames to get the time domain signal for the spatial average luminance of pixels. Next, we calculated the HVS response to this signal by using the discrete-time domain filter given in equation (6) and then, plotted the frequency domain representation of the output. We recorded the output of the LCD and the power consumption was recorded using a Data Acquisition System (DAQ).
Figure 6 compares the temporal behavior of the two different movie clips for different maximum allowed distortion rates. Note that the output of HEBS has a number of strong low frequency components, which cause unwanted flickering, whereas TABS has a frequency domain characteristic almost identical to the original sequence. Moreover, as we increase the maximum allowed video distortion the amplitude of different frequencies significantly increases for HEBS, whereas the amplitude of the TABS remains almost unchanged.

Figure 7 shows the overall energy savings of the LCD for different movie clips. HEBS achieves higher energy savings since it does not consider the temporal distortion. However, the loss in energy savings due to consideration of the temporal distortion in the TABS scheme is not significant whereas the overall video quality is significantly improved as depicted in Figure 6.

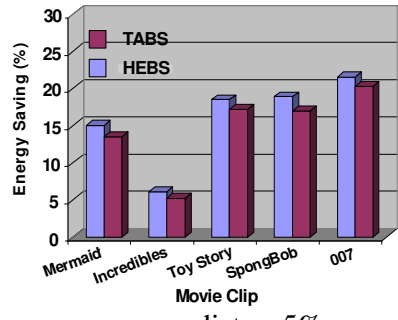

a. $\max$. dist. $=5 \%$

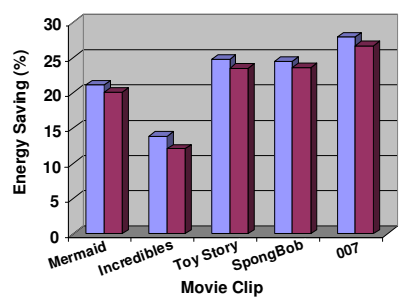

b. $\max$. dist. $=15 \%$
Figure 7. Energy savings - TABS vs. HEBS

\section{CONCLUSION}

We proposed a temporally-aware backlight scaling scheme for video, based on a detailed video distortion metric, which not only account for the intra-frame spatial distortion, but also uses the spectral power density of the average luminance of different frames to measure the flickering incurred by the backlight scaling. Experimental results show a significant improvement in quality of backlight scaled video.

\section{REFERENCES}

[1] N. Chang, et al., "DLS: Dynamic Backlight Luminance Scaling of Liquid Crystal Display," IEEE Transactions on VLSI Systems, Vol. 12, No. 8, Aug. 2004, pp.837-846.

[2] W-C. Cheng and M. Pedram, "Power Minimization in a Backlit TFT-LCD by Concurrent Brightness and Contrast Scaling," IEEE Trans. on Consumer Electronics, Vol. 50, No. 1, Feb. 2004, pp. 25-32.

[3] A. Iranli, et al., "HEBS: Histogram Equalization for Backlight Scaling," Proceedings of DATE, Feb. 2005.

[4] A. Iranli, M. Pedram, "DTM: Dynamic Tone Mapping for Backlight Scaling," Proceedings of DAC, Jun. 2005.

[5] N. Graham and D. Hood, "Modeling the dynamics of light adaptation: The merging of two traditions," Vision Res.32, 1373-93, 1992

[6] T.E. Wiegand, et al., "Testing a computational model of light-adaptation dynamics," Vision Res., 35(21), 3037-51, 1995.

[7] http://apollo.usc.edu/testbed/

[8] B.H. Crawford, "Visual adaptation in relation to brief conditioning stimuli," Proceedings of the Royal Society B 134, 283-302. 